\title{
A TRANSFERÊNCIA DE POLÍTICAS PÚBLICAS DE VIGILÂNCIA EPIDEMIOLÓGICA NO BRASIL E NA ARGENTINA'
}

\author{
POLICY TRANSFER FOR EPIDEMIOLOGICAL SURVEILLANCE IN BRAZIL \\ AND ARGENTINA
}

DOI: 10.5380/cg.v9i2.67858

\author{
Alexsandro Eugenio Pereira² \\ Victoria Silva Oliveira3
}

\begin{abstract}
Resumo
O presente artigo pretende identificar como ocorre o processo de transferência e difusão de políticas públicas no Mercosul, tendo como ênfase as normas aprovadas pelo Mercosul na área de Vigilância Epidemiológica. O objetivo principal é identificar normas dessa área e inferir sobre como elas são incorporadas por dois de seus Estados membros principais: Argentina e Brasil. Para desenvolver esse objetivo, o artigo identifica e examina as normas propostas no interior do Subgrupo de Trabalho $\mathrm{n}^{0} 11$ no que se refere ao temas da Vigilância Epidemiológica do Mercosul e, em seguida, aprovadas pelo Grupo do Mercado Comum (GMC), um dos principais órgãos decisórios do bloco. Em seguida, o artigo identificou como cada um dos Estados analisados incorporou as normas aprovadas por meio de resoluções do GMC e que se referem à vigilância epidemiológica. Os resultados alcançados apresentam uma classificação do status atual do processo de incorporação de cada uma dessas resoluções na Argentina e no Brasil. O Mercosul tem desafios políticos e econômicos a enfrentar, mas tem servido como um instrumento de transferência e difusão de políticas públicas, contribuindo em algumas áreas temáticas para a formulação de políticas públicas e arranjos normativos, como ocorreu na área de vigilância epidemiológica. Dessa forma, identificar o percentual de resoluções internalizadas pelos países membros contribui para destacar não só a complexidade dos estudos de transferência de políticas públicas como também para definir o grau em que as resoluções aprovadas são incorporadas pelos países membros.
\end{abstract}

Palavras-Chave: Difusão; Transferência de políticas públicas; Mercosul; Saúde.

\begin{abstract}
This article aims to identify how the process of transferring and disseminating public policies in Mercosur occurs, with emphasis on the rules approved by Mercosur in the area of Epidemiological Surveillance. The main objective is to identify norms in this area and to infer how they are incorporated by two of its main member states: Argentina and Brazil. In order to develop this objective, the article identifies and examines the rules proposed within Working Subgroup 11 regarding the themes of Mercosur Epidemiological Surveillance and subsequently approved by the Common Market Group (GMC), one of the main decision-making bodies of the bloc. Then, the article identified how each of the analyzed states incorporated the norms approved through GMC resolutions and which refer to epidemiological surveillance. The results achieved present a classification of the current status of the process of incorporating each of these resolutions in Argentina and Brazil. Mercosur has political and economic challenges to face, but it has served as an instrument for the transfer and diffusion of public policies, contributing in some thematic areas to the formulation of public policies and normative

\footnotetext{
${ }^{1}$ Este artigo foi originado de uma pesquisa financiada com recursos do CNPq (Conselho Nacional de Desenvolvimento Científico e Tecnológico).

${ }_{2}^{2}$ Doutor em Ciência Política pela Universidade de São Paulo (USP) e professor dos Programas de PósGraduação em Ciência Política e em Políticas Públicas da Universidade Federal do Paraná (UFPR). E-mail:

3 Mestranda em Desenvolvimento Econômico e graduada em Ciências Econômicas pela Universidade Federal do Paraná (UFPR); E-mail: whzpvictoria@gmail.com. ORCID: https://orcid.org/o000-0002-2771-7162.
} alexsep@uol.com.br; ORCID: https://orcid.org/0000-0002-9613-4702.
\end{abstract}


arrangements, as occurred in the area of epidemiological surveillance. In this way, identifying the percentage of resolutions internalized by member countries contributes to highlighting not only the complexity of public policy transfer studies, but also to defining the degree to which approved resolutions are incorporated by member countries.

Keywords: Diffusion; Policy Transfer; Mercosur; Health.

\section{INTRODUÇÃO}

O campo de transferência e difusão de políticas públicas é diversificado e de caráter multidisciplinar. A globalização e o rápido crescimento de diferentes meios de comunicação favorecem o intercâmbio de ideias, de conhecimentos e de políticas entre os países. Ao mesmo tempo, as instituições supranacionais e intergovernamentais de processos de integração regional se tornaram lócus importantes para a interação de atores políticos e sociais e possibilitaram uma interação que favorece a formulação de políticas, normas e resoluções regionais que são mais ou menos internalizadas pelos Estados-membros. Com isso, as organizações internacionais, entre as quais o Mercosul e a União Europeia, contribuem para favorecer processos de transferência e difusão de políticas públicas, ampliando a adoção de políticas similares por parte de um número de nações diferentes ao longo das últimas décadas (DOLOWITZ e MARSH, 2000).

Nesse sentido, o desenvolvimento de uma agenda de pesquisas empíricas sobre o tema é essencial para mensurar as possibilidades de transferência e difusão de políticas públicas a partir de organizações internacionais e de processos de integração regional. Para contribuir com esse desenvolvimento, autores como Dolowitz e Marsh (2000) sugerem uma tipologia que classifica a transferência de acordo com o grau em que a integralidade da política original é mantida pela unidade política receptora. Além disso, os autores indicam quais são os conteúdos que podem ser transferidos entre os países ou entre organizações internacionais e Estados nacionais. Segundo Dolowitz e Marsh (2000, p. 12), diferentes categorias de elementos podem ser transferidas como, por exemplo, objetivos políticos, conteúdos e instrumentos de políticas, programas políticos, instituições, ideologias, ideias e atitudes. Diane Stone (1999, p. 52) mencionou, também, que ferramentas regulatórias, administrativas ou judiciais podem ser adotadas por diferentes países para enfrentar determinados problemas específicos da agenda comum deles. É o que ocorre nos processos de integração regional e, em particular, na agenda de saúde do Mercosul, quando os países precisam definir estratégias comuns para lidar com os problemas da vigilância epidemiológica, com a circulação regional de produtos para a saúde e com a adoção de estratégias para permitir a circulação e o exercício de profissionais de saúde dentro dos Estados-Partes do Mercosul.

Neste artigo, o objetivo é analisar o tema da vigilância epidemiológica no Mercosul, com foco na Reunião de Ministros da Saúde, vinculada ao Conselho do Mercado Comum (CMC) e, sobretudo, no Subgrupo de Trabalho 11 da Saúde, subordinado ao Grupo do Mercado Comum (GMC). Seu propósito específico é avaliar como as resoluções aprovadas pelo GMC, no que se refere à vigilância 
epidemiológica, foram internalizadas por dois dos principais protagonistas da integração regional do Mercosul, Brasil e Argentina. A vigilância epidemiológica é um dos temas específicos da cooperação e das tentativas de articulação entre os Estados-Partes na área de saúde. Os esforços pela harmonização das políticas de saúde no bloco são realizados pelo SGT11. Esse Subgrupo foi criado em 1996 para buscar a harmonização das legislações dos Estados-Partes no que se refere "aos bens, serviços, matérias-primas e produtos da área da saúde, como também os critérios para a vigilância epidemiológica e o controle sanitário" (citado em MACHADO et. al., 2007, p. 295). Nesse sentido, uma parte da produção desse Subgrupo de Trabalho se apresenta na forma de propostas de resoluções, submetidas à aprovação do GMC. Num segundo momento, essas resoluções precisam ser internalizadas por meio de normas aprovadas em cada Estado Parte do Mercosul. Sem essa internalização, a cooperação na questão específica da vigilância epidemiológica não produz os resultados esperados. Sendo assim, a análise precisa se concentrar na internalização e o principal desafio de uma pesquisa dessa natureza é metodológico e envolve a

necessidade de percorrer a trajetória que se inicia na abordagem de determinado tema no Mercosul, nas reuniões especializadas e nos subgrupos de trabalho, com participação de representantes dos Executivos nacionais, e termina no espaço doméstico no qual são produzidas políticas públicas setoriais ou são internalizadas normas estabelecidas no âmbito regional (PEREIRA, 2015, p. 2-3).

Para serem internalizadas, as resoluções do GMC passam por um processo que envolve três momentos: harmonização nas comissões; internalização nos países; e implementação no Mercosul. A fase de internalização é a etapa mais demorada, considerando os ajustes necessários para incorporar as novas mudanças. Por isso, pode-se considerar que essa fase expressa o ritmo do processo de integração. Ao mesmo tempo, essa fase coloca em xeque a capacidade do Mercosul de produzir decisões capazes de ser incorporadas às políticas e às legislações dos Estados-Partes.

A coleta de dados da pesquisa que originou este artigo foi dividida em três etapas: a primeira procurou identificar os acordos aprovados pela Reunião de Ministros da Saúde, com base nas atas das reuniões das autoridades de saúde do Mercosul; a segunda buscou identificar todas as resoluções aprovadas pelo GMC e propostas pelo SGT 11 na área de Vigilância Epidemiológica no período de 1998 a 2019; e a terceira procurou definir o status de cada uma dessas resoluções no Brasil, na Argentina e no Mercosul. O processo de internalização de cada uma delas foi analisado individualmente no site internacional do Mercosul (www.mercosur.int) e nos respectivos ministérios da saúde do Brasil e da Argentina. Após a análise e a organização dos documentos do Mercosul e dos ministérios, utilizou-se uma classificação do status das resoluções em cada país. A classificação adotada foi a seguinte: i) incorporada; ii) não-incorporada; iii) revogada no Mercosul; iv) vigente no Mercosul; $v$ ) sem registro de vigência (quando não foi possível identificar informações sobre o status da resolução no Mercosul); vi) sem registro de incorporação (quando não foi possível identificar o status da resolução dentro do Estado Parte do Mercosul). 
O artigo está organizado em três seções, além dessa introdução e das considerações finais. A primeira realiza uma revisão bibliográfica acerca do tema, apresentando os principais conceitos sobre a transferência de políticas públicas. A segunda aborda a transferência e difusão de políticas públicas no Mercosul e destaca a importância do Brasil e da Argentina na integração do Mercosul, justificando a escolha desses dois países para o desenvolvimento da análise. Na seção seguinte, serão expostos os principais resultados do estudo realizado nas decisões do CMC, nas resoluções do GMC e na internalização dessas resoluções em cada país.

\section{A TRANSFERÊNCIA E DIFUSÃo DE POLÍticas PÚblicas: PRESSUPOSTOS TEÓRICOS}

Os primeiros estudos de transferência de políticas públicas possuíam um cunho mais sociológico que, ao longo do tempo, foram dividindo espaço com abordagens mais empíricas onde a globalização e o avanço nas telecomunicações possibilitaram o surgimento de fenômenos como a convergência, ou seja, políticas públicas cada vez mais parecidas entre os países. Autores como Dolowitz e Marsh (2000) preferem utilizar o termo policy transfer ou transferência de políticas públicas para designar esses fenômenos. Essa definição, manifestada pelos autores, consolida o termo como o:

conhecimento sobre políticas, arranjos administrativos, instituições e ideias em um cenário político (passado ou presente) é usado no desenvolvimento de políticas, arranjos administrativos, instituições e ideias em outro cenário político (DOLOWITZ e MARSH, 2000, p. 5; tradução livre)4.

O termo difusão, por sua vez, diferente da definição de transferência de políticas. Segundo Stone (2004), em termos de escala, a difusão pode ser entendida como a adoção de políticas por um grupo de países ou governos. Em outros casos, a difusão pode ser entendida como a adoção de uma política por um conjunto de países, que podem ser identificados por sua proximidade geográfica, como Estados latino-americanos. Sendo as abordagens de difusão importantes para analisar grupos de políticas adotadas, não é incomum que alguns analistas considerem o processo como um movimento linear em que as políticas se espalham por diferentes países em um processo mecânico (OLIVEIRA e FARIA, 2017).

O termo convergência, por sua vez, envolve o aumento na similaridade das políticas públicas adotadas pelos países ao longo do tempo e é também um conceito chave na literatura que estuda políticas públicas. Assim, enquanto a transferência se refere à disseminação consciente de políticas

\footnotetext{
4 Texto original: "[...] knowledge about policies, administrative arrangements, institutions and ideas in one political system (past or present) is used in the development of policies, administrative arrangements, institutions and ideas in another political system".
} 
e ideias entre países, a convergência não é necessariamente um resultado da transferência de políticas (STONE, 2004). O processo de lesson-drawing apresentado por Dollowitz (2017) é apontado nesse contexto, visto que esse autor está interessado, também, no modo como as ideias e as políticas se espalham. Porém, ao contrário da difusão, lesson-drawing direciona sua atenção aos microprocessos envolvidos no movimento dessas ideias e políticas em todos os limites geográficos.

Formulações aprofundadas sobre o tema puderam ser cada vez mais exploradas através dessas definições. Autores como Bulmer e Padgett (2004) relacionaram, por exemplo, estes variados tipos de transferência a arranjos institucionais. Dessa forma, conseguiram apresentar três formas distintivas de governança (hierarquia, negociação e facilitação) e as contextualizaram no cenário da União Europeia (UE). Portanto, segundo eles (2007, p.104; tradução livre):

A governança hierárquica prevalece em áreas políticas como o mercado único, onde as instituições da UE exercem autoridade supranacional levando a formas coercivas de transferência. Uma segunda forma de governança ocorre onde a União Europeia procura concordar com regras ou normas por consentimento comum (ou maioria). Não é incomum encontrar normas da UE modeladas sobre um ou mais Estados membros em uma forma de transferência por negociação. Finalmente, onde os Estados membros mantêm a soberania, mas coordenam a política através das instituições da UE (como na Justiça e Assuntos Internos), a transferência de políticas assumirá a forma de troca unilateral voluntária facilitada pela União Europeia5.

Além desta, outra manifestação sugerida foi a separação dos tipos de grau de transferência de políticas públicas. Tal separação foi proposta por Dollowitz e Marsh (200o, p. 13), que sinalizam para quatro diferentes de graus de transferência, a saber:

cópia, que envolve transferência direta e completa; emulação, que envolve a transferência das ideias por trás da política ou programa; combinações, que envolvem misturas de várias políticas diferentes; e inspiração, onde a política em outra jurisdição pode inspirar uma mudança de política mas o resultado final não se baseia no original (DOLOWITZ e MARSH, 2000, p. 13; tradução livre) ${ }^{6}$.

Dollowitz e Marsh (2000) classificam os diferentes tipos de transferência ao longo de um continuum que representa uma sequência na qual os elementos adjacentes não são perceptivamente diferentes uns dos outros, embora os extremos sejam bastante distintos. Nesse continuum, os extremos são representados por dois processos: o processo de lesson-drawing; e, no outro extremo,

\footnotetext{
5 Texto original: "Hierarchical governance is prevalent in policy areas like the single market, where EU institutions exercise supranational authority leading to coercive forms of transfer. A second form of governance occurs where the European Union seeks to agree common rules or norms by common (or majority) consent. It is not uncommon to find EU norms modelled on those of one or more member state(s) in a form of transfer by negotiation. Finally, where member states retain sovereignty but co-ordinate policy via EU institutions (as in Justice and Home Affairs), policy transfer will take the form of unilateral, voluntary exchange facilitated by the European Union".

${ }^{6}$ Texto original: "copying, which involves direct and complete transfer; emulation, which involves transfer of the ideas behind the policy or program; combinations, which involve mixtures of several different policies; and inspiration, where policy in another jurisdiction may inspire a policy change, but where the final outcome does not actually draw upon the original".
} 
o processo de transferência coercitiva (transferência como resultado de obrigações de tratados, etc.). À vista disso, os pesquisadores puderam capturar sutilezas envolvidas na transferência, como se a mesma permanecesse voluntária ou se transformasse em um processo coercitivo ao longo do tempo, ou mesmo variado entre diferentes unidades políticas dentro do mesmo sistema político. A Figura 1, a seguir, sintetiza o continuum mencionado acima.

FIGURA I - DE LESSON-DRAWING PARA TRANSFERÊNCIA COERCIVA

\begin{tabular}{|c|c|c|}
\hline \multicolumn{3}{|c|}{ Obligated Transfer (transfer as a result of treaty obligations, etc.) } \\
\hline $\begin{array}{l}\text { Lesson-Drawing } \longleftarrow \\
\text { (perfect rationality) }\end{array}$ & & $\longrightarrow \rightarrow \begin{array}{l}\text { Coercive Transfer } \\
\text { (direct imposition) }\end{array}$ \\
\hline $\begin{array}{l}\text { Lesson-drawing } \\
\text { (bounded rationality) }\end{array}$ & $\begin{array}{l}\text { Voluntarily } \\
\text { but driven by perceived } \\
\text { necessity (such as the } \\
\text { desire for international } \\
\text { acceptance) }\end{array}$ & Conditionality \\
\hline
\end{tabular}

Fonte: Dollowitz e Marsh, 2000, p.13.

Este processo coercitivo pode, também, ser dividido em cinco: pela indução, levando os governos a promulgar políticas que de outra forma não teriam iniciado; seletividade, auxílio dado apenas aos países que já apresentam um "bom" ambiente de política; paternalismo, onde os doadores tentam obter bens e serviços que eles favorecem; e pela restrição, buscando proteção contra a reversão da política que pode ocorrer com um novo governo. Essa forma difere da indução, na medida em que não há desacordo político entre o país receptor e o doador; e, por último, a sinalização, onde a ajuda é usada como um dispositivo para sinalizar o comportamento de "boa" política pelo país destinatário (STONE, 2004).

Temos, então, organizações internacionais, dentre outras, utilizando-se principalmente da coerção para promover "melhores práticas" ou adesão aos padrões internacionais. Ou seja, diferentemente das organizações governamentais, elas buscam meios para ajudar a desenvolver respostas políticas comuns em alguns campos. UNAIDS (Programa Conjunto das Nações Unidas sobre HIV/Aids), por exemplo, é uma iniciativa multilateral para coordenar as respostas a esta pandemia. A União Europeia (UE) é um exemplo, também, de instituição que promove a convergência dos Estados (STONE, 2004). Isso posto, é possível inferir que o fenômeno da transferência em organizações intergovernamentais é mais provável de ocorrer quando há uma jurisdição geográfica, ideológica ou culturalmente próxima. Nesse sentido, o Mercado Comum do Sul (Mercosul) se mostra propício para o estudo desses fenômenos.

O processo de transferência torna os formuladores de políticas, nos níveis nacionais e internacionais, dependentes de um conselho de consultores, sejam eles indivíduos ou empresas. Essa 
dependência torna mais complicada a definição do que seria uma transferência voluntária ou coercitiva. Por exemplo, os consultores podem "impor" um modelo de reforma do mercado aos países, sejam eles contratados por um governo, representantes de uma agência de ajuda internacional ou, ainda, atuando de forma independente. Tal situação claramente tem elementos de transferência voluntária e coercitiva.

Em síntese, analisar a transferência de políticas públicas é essencial no interior de organizações internacionais, como o Mercosul e a União Europeia. No contexto da globalização, a transferência tornou-se crescente, pois, conforme mostrou Stone (1999, p. 5), alguns governos e OIs buscam harmonização em determinados temas da agenda de políticas públicas, como será possível observar na análise da questão da Vigilância Epidemiológica no Mercosul na terceira seção deste artigo. Antes, na próxima seção, será examinada como a transferência de políticas vem ocorrendo no processo de integração do Mercosul mais recentemente e a importância do Brasil e da Argentina nesse processo.

\section{TRANSFERÊNCIA de POLÍticas PÚblicas NO MERCOSUl E O PAPEL DO BRASIL E DA ARGENTINA NO PROCESSO DE INTEGRAÇÃO REGIONAL}

Desde 1991, ano de sua fundação, o Mercosul tem funcionado com base em seus mecanismos intergovernamentais e no processo de tomada de decisão fundado no consenso. Frequentemente, comparações com outros blocos regionais, como a União Europeia, são feitas na tentativa, dentre outras, de entender melhor a organização mercosulina (MEDEIROS; MEUNIER; COCKLES, 2015). Se no bloco europeu houve, gradualmente, a implementação de instituições com caráter supranacional, no Mercosul tal fato não ocorreu. Dessa forma, as decisões continuam concentradas nos dois principais órgãos decisórios do bloco (GMC e CMC). No que se refere à sua agenda, o bloco privilegiou, em seus momentos iniciais, as relações comerciais. Seguindo um panorama histórico, essas relações sofreriam mudanças apenas após a segunda metade da década de 1990, com o surgimento do "Mercosul Social" onde se manifesta, então, um interesse em pensar estratégias de harmonização no campo das políticas sociais do bloco (MARIN, 2011).

Um dos exemplos dessas estratégias, segundo Marin (2011), pode ser observada na Reunião Especializada sobre Agricultura Familiar (REAF), cujos objetivos são: "fortalecer as políticas públicas para a agricultura familiar no Mercosul e promover e facilitar o comércio da produção de base familiar, a partir dos princípios da solidariedade e da complementaridade, visando à redução das assimetrias e o desenvolvimento da região" (REAF, citada em MARIN, 2011, p.19). De fato, a REAF mostrou ser um espaço com potencial de transferência de políticas públicas. Um dos exemplos, foi a adoção, por parte da Argentina e do Uruguai, da temática da "agricultura familiar”, conceito este oriundo do Brasil. 
Apesar disso, é inegável que o baixo grau de autoridade conferido às instituições no Mercosul dificulta o avanço em diversos temas da agenda do chamado "Mercosul Social". A lógica estabelecida dentro do Mercosul aponta para uma governança por facilitação, nos termos de Bulmer e Padgett, citados na seção precedente deste artigo. Essa forma de governança favorece a transferência de políticas públicas, conforme mostraram as pesquisas desenvolvidas por Marin (2011) e por Pereira et al. (2018).

Mas é preciso notar que o processo de integração do Mercosul foi resultado não somente do grau de convergência entre os quatro países-membros fundadores do bloco econômico, mas, também, da evolução do Brasil e da Argentina que já desempenhavam o protagonismo ao promover precocemente um processo de aproximação e cooperação envolvendo iniciativas nos planos político, econômico e estratégico.

Esse argumento pode ser sustentado a partir de uma breve contextualização histórica. Na década de 1980, durante o período de redemocratização, as políticas externas de ambos os países já encontravam elementos de convergência. Se de um lado a Argentina do governo Alfonsín (19831989) buscava recuperar sua credibilidade externa deteriorada, dentre outras coisas, devido à Guerra das Malvinas, o Brasil do governo Sarney (1985-1990) enfrentava o desafio de adaptar sua política externa às novas condições do cenário internacional. Segundo Vaz (2002, p.74), "para o Brasil, preservava-se o sentido universalista de sua política exterior, mas dentro dele, foi fortalecida e ampliada a prioridade às relações com a América Latina, que passaram a encontrar, finalmente sentido de funcionalidade no plano econômico”.

Logo, a partir de 1979, há a efetivação da construção da parceria Brasil-Argentina com a solução da questão da Itaipu-Corpus que, em 1985, pôs fim ao problema da utilização dos recursos hídricos no trecho do Rio Paraná e seria impulsionada com a Ata Iguaçu, na qual os dois países se mostravam dispostos a acelerar o processo de integração bilateral. Na década de 1990, aumentou a convergência entre os dois países. Isto é, a relação entre eles representaria agora um espaço e oportunidade de aprendizado e adaptação dos setores privados para a abertura e para a exposição à concorrência externa, representando principalmente uma resposta adaptativa à formação de blocos econômicos. O ano de 1991 representa, portanto, o auge do processo de integração entre Brasil e Argentina, pois, com o Tratado de Assunção entre os dois países, acrescidos do Paraguai e Uruguai inicialmente, ocorre a criação do Mercosul, no qual as agendas econômicas domésticas se vinculariam às novas tendências da economia mundial de forma a permitir que os países membros negociassem conjuntamente sua inserção internacional (VAZ, 2002).

No final dos anos 1990, o estágio do processo de integração não alcançou o que foi previsto no Tratado de Assunção. Alternou períodos de avanços com outros de certa paralisia, de acordo com as conjunturas econômicas e os contextos políticos (SARAIVA et al, 1999). Os momentos de paralisia geraram dúvidas sobre os ganhos econômicos e políticos possíveis do processo de integração e colocaram em risco a continuidade desse processo. Os conflitos comerciais e as consequentes 
dificuldades de relacionamento entre Brasil e Argentina foram essenciais para se compreender a dinâmica da integração no Mercosul. O mesmo ocorreu nos anos 2000, quando uma convergência política entre os governos brasileiro (com a eleição do presidente Luiz Inácio Lula da Silva) e argentino (com Nestor Kirchner), favoreceu o desenvolvimento de uma agenda social no bloco, ao lado dos temas comerciais que predominaram no Mercosul, após sua criação em 1991.

Sendo assim, considerando os limites deste artigo, faz sentido concentrar a análise nesses dois protagonistas do processo de integração do Mercosul, sem desconsiderar a possibilidade de uma análise sobre os demais Estados-Partes no que se refere à agenda social, de modo geral, ou à temática de saúde, em particular. Neste artigo, a opção foi pelo estudo da incorporação das normas emanadas do GMC no Brasil e na Argentina para efeitos de análise. Os dados desse estudo são apresentados e discutidos na próxima seção deste artigo.

\section{AS POLÍTICAS DE VIGILÂNCIA EPIDEMIOLÓGICA: METODOLOGIA, ANÁLISE E RESULTADOS}

Desde 1994, com o Protocolo de Ouro Preto, foram acrescidos outros 53 ao Tratado de Assunção. A partir daí o bloco adquiriu uma nova relevância ao consolidar, dentre outras coisas, o sistema multilateral de comércio. O Protocolo de Ouro Preto estabeleceu, também, a estrutura institucional do bloco econômico. Nele, há a descrição do processo de institucionalização das normas. O seu artigo $42^{\circ}$ dispõe que:

\footnotetext{
as normas emanadas dos órgãos do Mercosul previstos no Artigo 2 deste Protocolo terão caráter obrigatório e deverão, quando necessário, ser incorporadas aos ordenamentos jurídicos nacionais mediante os procedimentos previstos pela legislação de cada país. (disponível em: MERCOSUL, 2019a; tinyurl.com/y38otxrl; Acesso em: 30/10/2019).
}

Atualmente a estrutura institucional do Mercosul é composta por diversos foros de negociação, entre reuniões, comissões e subcomissões. Porém, segundo o Protocolo de Ouro Preto, o Mercosul possui três órgãos decisórios principais, de natureza intergovernamental: o Conselho do Mercado Comum, formado pelos ministros das Relações Exteriores e da Economia dos EstadosPartes; o Grupo do Mercado Comum, formado por quatro membros titulares e quatro membros alternos por país, indicados pelos governos nacionais, sendo que eles devem ser representantes dos ministérios das Relações Exteriores, da Economia e dos Bancos Centrais dos Estados-Partes; e a Comissão de Comércio do Mercosul, composta por quatro integrantes titulares e quatro alternos de cada Estado Parte, sendo coordenada pelos Ministérios das Relações Exteriores dos países.

Dentro dessa estrutura, foram identificados os arranjos relativos à institucionalidade da saúde. A temática da saúde ganhou impulso após o surgimento de foros específicos: a Reunião dos 
Ministros da Saúde (RMS), criada em 1995 e subordinada ao Conselho do Mercado Comum. Sua função é propor ao CMC medidas destinadas à coordenação de políticas na área de saúde; o Subgrupo de Trabalho $n^{0} 11$ (SGT 11), criado pela Resolução GMC nº 151/1996, é um órgão técnico subordinado ao Grupo Mercado Comum (GMC). O SGT 11 está dividido, ainda, em três áreas de trabalho: Vigilância em Saúde, Produtos para Saúde; e Serviços de Atenção à Saúde. Cada uma dessas áreas, conta com diferentes subcomissões e grupos ad hoc. A Figura 2 abaixo sintetiza o organograma do Mercosul a partir do qual é possível localizar os foros específicos que tratam da questão da saúde no bloco:

FIGURA II - ORGANOGRAMA MERCOSUL

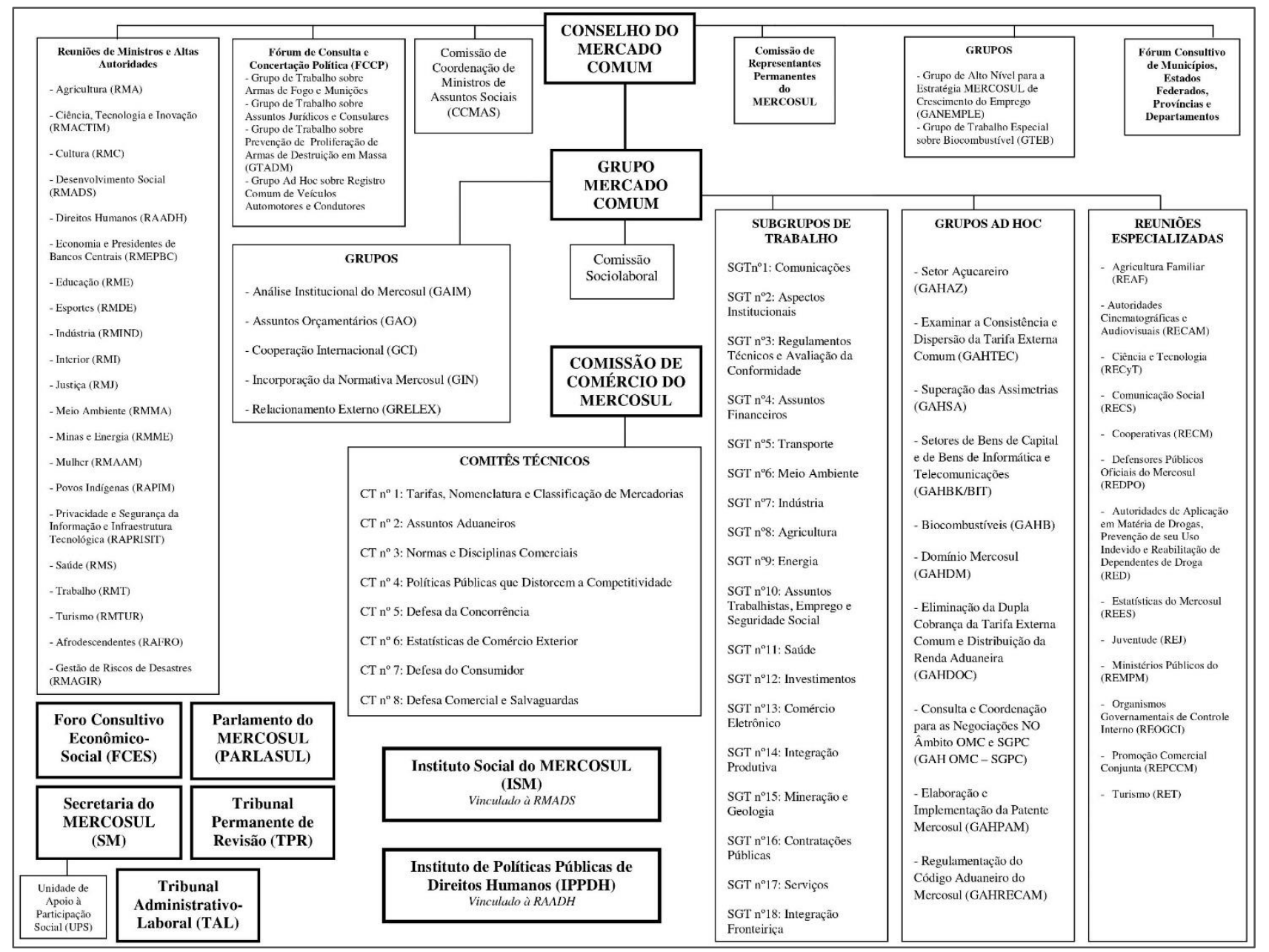

Fonte: MERCOSUL, 2019b.

Na Figura 2, nota-se que a Reunião de Ministros da Saúde (RMS) está localizada no lado esquerdo, pois está vinculada ao Conselho do Mercado Comum. Os resultados da RMS aparecem na forma de acordos regionais sobre diversos temas da agenda de saúde do Mercosul. Na área de vigilância epidemiológica, observa-se que a dengue é o tema com o maior número de acordos visto que, devido às condições climáticas, o território das Américas favorece a proliferação do mosquito 
transmissor, o Aedes aegypti. Esse foco no combate à dengue foi intensificado, também, com a criação do Plano Regional de Combate à Dengue, através do Acordo nº 14/2004.

É possível notar, também, que a disseminação do HIV/AIDS representa preocupação constante das autoridades de saúde dos Estados-Partes, sendo o segundo tema com maior número de acordos aprovados regionalmente. Esses acordos são fundamentais para promover a atenção sobre temas que demandam cooperação dos Estados e ações e planos integrados, pois o combate à dengue, por exemplo, possui relevância regional. O sucesso dessas ações de combate e prevenção à dengue ou ao HIV/AIDS implica o comprometimento dos países do Mercosul, pois a natureza desses temas exige elaboração e execução de estratégias comuns entre os Estados-Partes, com o propósito de lidar com doenças que podem afetar as populações dos diferentes países do Cone Sul e da Venezuela. O Quadro 1, a seguir, apresenta uma síntese dos 43 acordos aprovados no período de 1998 a 2019 dentro da RMS, revelando como o tema da vigilância epidemiológica esteve presente nas reuniões das autoridades de saúde do Mercosul. Não foram localizados acordos nos anos de 2016 e 2019.

QUADRO I - ACORDOS RMS SOBRE VIGILÂNCIA EPIDEMIOLÓGICA (1998 - 2019) [continua]

\begin{tabular}{|c|c|l|}
\hline ANO & $\begin{array}{c}\text { NUEERODE } \\
\text { ACORDOS } \\
\text { APROVADOS }\end{array}$ & $\begin{array}{l}\text { Controle da doença de Chagas } \\
\text { Dengue } \\
\text { Erradicação do Sarampo } \\
\text { Comissão de Preparativos para Desastres }\end{array}$ \\
\hline 1998 & 05 & $\begin{array}{l}\text { Acordo e políticas de combate à Dengue } \\
\text { Reconhecimento da cólera como objeto de vigilância epidemiológica } \\
\text { Acordo e políticas de combate ao Sarampo } \\
\text { Informes epidemiológicos e reuniões de técnicos } \\
\text { Verificação de água potável } \\
\text { Promoção de ações de controle de veículos de transporte terrestre, marítimo e aéreo }\end{array}$ \\
\hline 1999 & 07 & Prevenção e Controle da Dengue \\
\hline 2002 & 01 & $\begin{array}{l}\text { Enfermidades que podem ser evitadas por meio de vacinação } \\
\text { Prevenção e Controle da Dengue } \\
\text { Capacitação de profissionais de saúde } \\
\text { Controle do HIV/AIDS }\end{array}$ \\
\hline 2003 & 06 & $\begin{array}{l}\text { Prevenção e Controle da Dengue } \\
\text { Redução da transmissão infantil do HIV e da Sífilis Congênita }\end{array}$ \\
\hline 2005 & 02 & $\begin{array}{l}\text { Prevenção e Controle da Dengue } \\
\text { Enfrentamento de eventual pandemia de influenza e gripe aviária }\end{array}$ \\
\hline 2006 & 01 & Entendimento dos ministros para a luta contra o HIV/AIDS \\
\hline 2007 & 02 & Prevenção e Controle da Dengue \\
\hline 2008 & 03 & $\begin{array}{l}\text { Prevenção e Controle da febre amarela } \\
\text { Plano integrado para prevenção contra pandemia de influenza } \\
\text { Intensificação de ações para prevenção do HIV/AIDS }\end{array}$ \\
\hline 2009 & 01 & Prevenção e Controle da Dengue \\
\hline 2011 & 01 & Prevenção para evitar transmissão infantil do HIV/AIDS e da Sífilis \\
\hline 2012 & 05 & $\begin{array}{l}\text { Controle da tuberculose } \\
\text { Prevenção e Controle da Dengue } \\
\text { Prevenção e Controle da Leishmaniose } \\
\text { Aç̃̃es de saúde em HIV/AIDS para pessoas privadas de liberdade } \\
\text { Aç̃̃es de prevenção, atenção e apoio em HIV/AIDS }\end{array}$ \\
\hline
\end{tabular}


QUADRO I - ACORDOS RMS SOBRE VIGILÂNCIA EPIDEMIOLÓGICA (1998 - 2019) [conclusão]

\begin{tabular}{|c|c|l|}
\hline ANO & $\begin{array}{c}\text { NUMERODE } \\
\text { ACORDOS } \\
\text { APROVADOS }\end{array}$ & \multicolumn{1}{c|}{ TEMAS } \\
\hline 2013 & 02 & $\begin{array}{l}\text { Prevenção e Controle da Dengue } \\
\text { Controle da Tuberculose }\end{array}$ \\
\hline 2015 & 01 & Ações de controle da epidemia de HIV/AIDS \\
\hline 2017 & 01 & $\begin{array}{l}\text { Cooperação técnica para fortalecer cumprimento do Regramento Sanitário } \\
\text { Internacional na região }\end{array}$ \\
\hline 2018 & 01 & Fortalecimento das autoridades sanitárias reguladoras no Mercosul \\
\hline TOTAL & 43 & \\
\hline
\end{tabular}

Fonte: Elaborado pelos autores com base nos Acordos da Reunião de Ministros da Saúde (RMS). MERCOSUL, 2019c.

A Vigilância Epidemiológica representa, portanto, um instrumento relevante (i) ao apresentar o caráter e os riscos epidemiológicos e (ii) ao expor os dados sobre a presença das doenças em cada região. A Figura 3, a seguir, destaca os temas que despertaram maior interesse das autoridades de saúde do Mercosul.

\section{FIGURA III - TEMAS DOS ACORDOS DA RMS SOBRE VIGILÂNCIA EPIDEMIOLÓGICA}

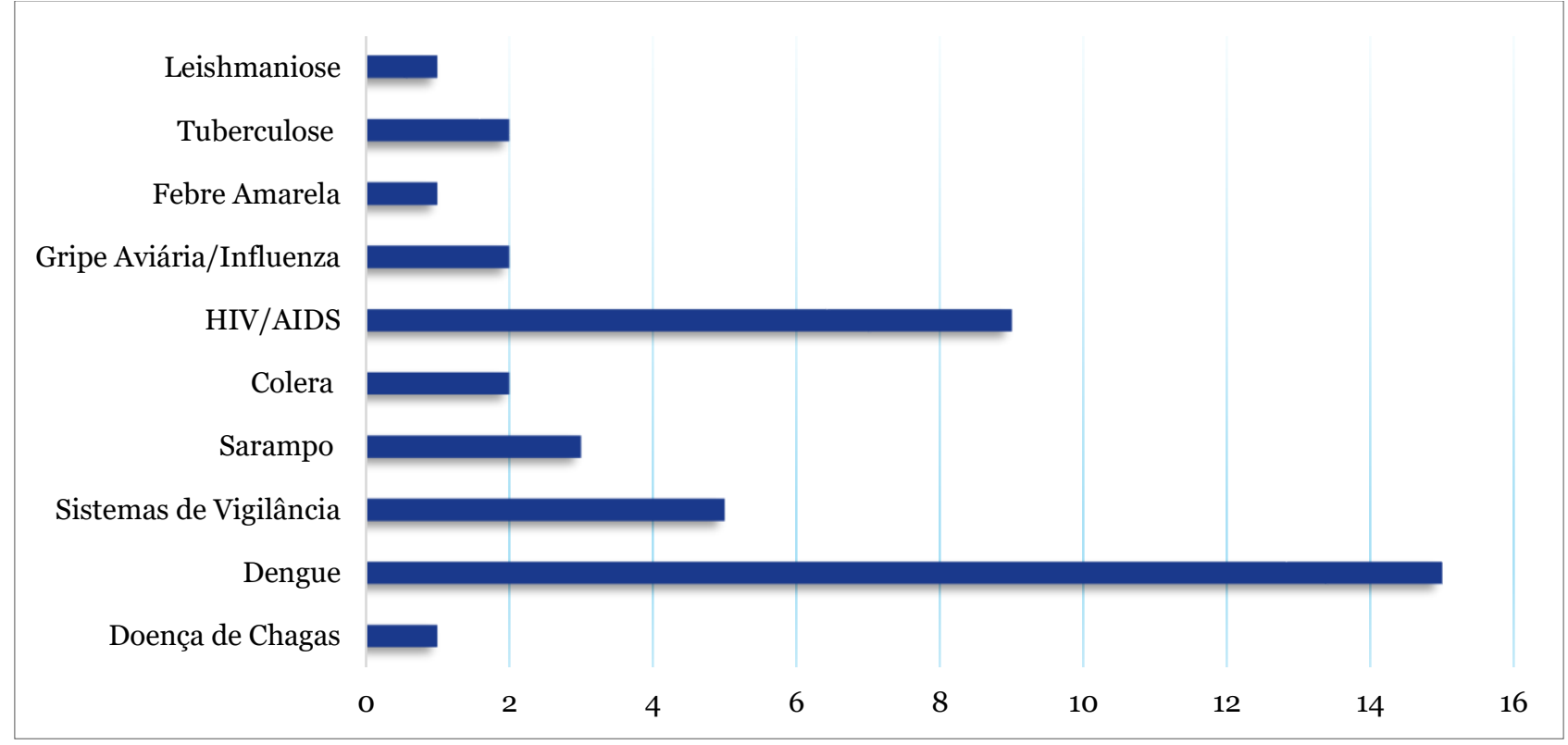

Fonte: Elaborado pelos autores com base nos Acordos da Reunião de Ministros da Saúde (RMS). MERCOSUL, 2019c.

As decisões aprovadas pelos Estados envolvem ações e planos integrados, com a participação e o envolvimento de todos os Estados. Na área de trabalho do SGT 11 Saúde, por sua vez, as resoluções aprovadas no GMC precisam ser executadas pelos Estados-Partes, o que implicaria certo grau de comprometimento dos governos nacionais e dos seus respectivos ministérios da saúde. A pesquisa que originou este artigo se concentrou, sobretudo, nessas resoluções e no seu processo de internalização ou incorporação. 
Com base na classificação apresentada na introdução deste artigo, o Quadro 2 abaixo apresenta: (i) as resoluções aprovadas pelo GMC, oriundas do SGT 11 da área de Vigilância Epidemiológica; e (ii) o status de incorporação dessas resoluções no Brasil e na Argentina, bem como sua atual vigência no bloco. O período pesquisado teve início em 1999 e terminou em 2019. Porém, depois de 2015, não foram localizadas novas resoluções do GMC sobre a temática de vigilância epidemiológica. Os anos de 2004, 2010 e 2014 não tiveram, também, resoluções aprovadas nessa temática específica. No total, foram identificadas 45 resoluções. Desse total, 9 resoluções foram aprovadas para revogar outras, publicadas em anos anteriores. Por isso, elas não foram classificadas e não fazem parte dos dados que serão apresentados no Quadro 2 abaixo, que contém a classificação de 36 resoluções do GMC.

QUADRO II - RESOLUÇÕES PROPOSTAS PELO GMC PROVENIENTES DO SGT 11 NA ÁREA DE VIGILÂNCIA EPIDEMIOLÓGICA (1999 - 2019)

\begin{tabular}{|c|c|c|c|c|}
\hline$A N O$ & $\begin{array}{l}\text { NÚMERO DE } \\
\text { RESOLUÇÕES }\end{array}$ & STATUS NO BRASIL & STATUS NA ARGENTINA & $\begin{array}{l}\text { VIGÊNCIA NO } \\
\text { MERCOSUL }\end{array}$ \\
\hline 1999 & 03 & $\begin{array}{l}1 \text { incorporada } \\
2 \text { não-incorporadas }\end{array}$ & $\begin{array}{l}2 \text { incorporadas } \\
1 \text { não-incorporada }\end{array}$ & 3 revogadas \\
\hline 2000 & 04 & $\begin{array}{l}1 \text { incorporada } \\
3 \text { não-incorporadas }\end{array}$ & $\begin{array}{l}1 \text { incorporada } \\
3 \text { não-incorporadas }\end{array}$ & 4 revogadas \\
\hline 2001 & 02 & $\begin{array}{l}1 \text { sem registro de } \\
\text { incorporação } \\
1 \text { incorporada }\end{array}$ & $\begin{array}{l}1 \text { sem registro de } \\
\text { incorporação } \\
1 \text { incorporada }\end{array}$ & $\begin{array}{l}1 \text { Sem registro de } \\
\text { incorporação } \\
1 \text { revogada }\end{array}$ \\
\hline 2002 & 04 & $\begin{array}{l}3 \text { incorporadas } \\
1 \text { não-incorporada }\end{array}$ & $\begin{array}{l}1 \text { incorporada } \\
3 \text { não-incorporadas }\end{array}$ & 4 revogadas \\
\hline 2003 & 05 & 5 incorporadas & $\begin{array}{l}2 \text { incorporadas } \\
3 \text { não-incorporadas }\end{array}$ & $\begin{array}{l}1 \text { Sem registro de } \\
\text { incorporação } \\
2 \text { revogadas } \\
2 \text { vigentes }\end{array}$ \\
\hline 2005 & 04 & $\begin{array}{l}1 \text { sem registro de } \\
\text { incorporação } \\
3 \text { incorporadas }\end{array}$ & $\begin{array}{l}1 \text { sem registro de } \\
\text { incorporação } \\
3 \text { incorporadas }\end{array}$ & $\begin{array}{l}3 \text { vigentes } \\
1 \text { sem registro de vigência }\end{array}$ \\
\hline 2006 & 01 & não-incorporada & incorporada & revogada \\
\hline 2007 & 02 & $\begin{array}{l}2 \text { não requerem } \\
\text { incorporação }\end{array}$ & $\begin{array}{l}2 \text { não requerem } \\
\text { incorporação }\end{array}$ & 2 revogadas \\
\hline 2008 & 05 & 5 incorporadas & 5 incorporadas & $\begin{array}{l}3 \text { sem registro de vigência } \\
1 \text { vigente } \\
1 \text { revogada }\end{array}$ \\
\hline 2009 & 02 & $\begin{array}{l}1 \text { incorporada } \\
1 \text { não-incorporada }\end{array}$ & 2 incorporadas & $\begin{array}{l}1 \text { vigente } \\
1 \text { sem registro de vigência }\end{array}$ \\
\hline 2011 & 01 & não-incorporada & incorporada & sem registro de vigência \\
\hline 2013 & 01 & não-incorporada & incorporada & sem registro de vigência \\
\hline 2015 & 02 & $\begin{array}{l}1 \text { não-incorporada } \\
1 \text { não requer incorporação }\end{array}$ & $\begin{array}{l}1 \text { incorporada } \\
1 \text { não requer incorporação }\end{array}$ & $\begin{array}{l}1 \text { sem registro de vigência } \\
1 \text { vigente }\end{array}$ \\
\hline TOTAL & 36 & & & \\
\hline
\end{tabular}

Fonte: Elaborado pelos autores com base nas resoluções do GMC. MERCOSUL, 2019d.

A partir dos dados do Quadro 2 acima, o Quadro 3 sistematiza o status das resoluções do GMC no Brasil e na Argentina, considerando que algumas resoluções não requerem incorporação. Há casos de resoluções sem registros sobre sua incorporação em cada um dos países. 
QUADRO III - STATUS DAS RESOLUÇÕES DO GMC NA ÁREA DE VIGILÂNCIA EPIDEMIOLÓGICA NO BRASIL E NA ARGENTINA (1999 - 2019)

\begin{tabular}{|c|c|c|}
\hline STATUS & BRASIL & ARGENTINA \\
\hline Incorporadas & 20 & 10 \\
\hline Não-incorporadas & 11 & 03 \\
\hline Não requerem incorporação & 03 & 02 \\
\hline Sem registro de incorporação & 02 & 36 \\
\hline TOTAL & 36 & 21 \\
\hline
\end{tabular}

Fonte: Elaborado pelos autores com base nas resoluções do GMC. MERCOSUL, 2019d.

É preciso notar que há um número significativo de resoluções revogadas - 18 das 36 resoluções. Outras 10 não têm registro de vigência enquanto 08 das 36 resoluções estão vigentes. Para efeitos de análise, optou-se por considerar o conjunto das 36 resoluções, que apresentava decisões específicas na área de vigilância epidemiológica. Desse total, o Brasil incorporou 20 das 36 resoluções, como se pode observar no Quadro 3 acima. Na Argentina, por sua vez, observou-se que 21 das 36 resoluções foram incorporadas. Os números de cada país e apresentados no Quadro 3 acima são, no geral, bastante similares, o que demonstra uma proximidade dos percentuais de internalização das normas entre os dois países. Na Argentina, foram incorporadas 58,35\% das resoluções do GMC. No Brasil, 55,56\% dessas resoluções foram incorporadas.

Os dados mostram que há redução do número de resoluções do GMC, específicas sobre a área de Vigilância Epidemiológica, sobretudo se forem considerados os últimos 4 anos - de 2016 a 2019. No entanto, as resoluções propostas obtiveram certo grau de internalização - superior a 50\% -, isto é, existem mais normas vigentes ao longo dos anos. A natureza dos temas tratados nas reuniões do SGT11 pode explicar os motivos pelos quais há relativo interesse dos Estados na internalização. São temas que demandam esforços de cooperação e colaboração técnica. Além disso, as reuniões das autoridades de saúde dos países do Mercosul na RMS e as atividades de compartilhamento - de ideias, conhecimentos, dados e informações sobre os temas da vigilância epidemiológica - no SGT11 sugerem que o Mercosul tem contribuído como espaço de facilitação para a construção de decisões regionais (RMS) ou de normas de vigilância epidemiológica (GMC) que são, posteriormente, ratificadas internamente.

O grau de internalização dessas normas poderia ser mais expressivo, quando se supõe que as resoluções do GMC são aprovadas pelos representantes dos governos nacionais e, nesse sentido, expressam a vontade desses governos. Além disso, segundo o Protocolo de Ouro Preto, as normas aprovadas pelos órgãos decisórios do Mercosul precisam ser incorporadas pelos Estados-Partes, de acordo com o compromisso firmado por eles nesse Protocolo e no Tratado de Assunção. Porém, é preciso reconhecer que a baixa institucionalização do Mercosul, adotada como opção pelos governos dos Estados-Partes (ver VIGEVANI et al., 2008), limita a possibilidade de se efetuar sempre uma transferência do tipo coercitiva, conforme sugeriu a abordagem de Dolowitz e Marsh, apresentada na primeira seção deste artigo. É evidente que outras variáveis também influenciam o processo de 
transferência na área específica da vigilância epidemiológica, como a dinâmica burocrática dos ministérios da saúde dos dois países, responsáveis pela internalização das resoluções do GMC. Mas as características institucionais vigentes no Mercosul continuam sendo elemento importante na análise dos resultados da transferência em áreas temáticas específicas. Conforme se mostrou em outro artigo (ver PEREIRA et al., 2018), o Mercosul funciona nos termos do que Bulmer e Padgett chamaram de governança facilitada, própria de um processo de integração com baixa institucionalização.

Em síntese, conforme apontado na introdução deste artigo, a agenda de saúde no Mercosul envolve, sobretudo, a transferência de ferramentas regulatórias e administrativas que podem ser, mais ou menos, adotadas pelos diferentes Estados-Partes do Mercosul. É a forma como eles pretendem lidar com determinados problemas de sua agenda comum. Na agenda decisória da vigilância epidemiológica no Mercosul tem prevalecido o debate e a produção de normas e recomendações de natureza técnica. Por isso, verifica-se, com maior predominância, a transferência dessas normas, capazes de estabelecer, minimamente, a condução governamental de assuntos relativos à vigilância epidemiológica. Entretanto, mesmo a análise dessas normas permite construir inferências sobre as possibilidades do Mercosul no que se refere à policy transfer, do espaço regional para o interior de cada Estado Parte. É o que foi possível verificar na análise específica realizada neste artigo.

\section{CONSIDERAÇÕES FINAIS}

A partir da análise realizada neste artigo, foi possível identificar os principais pressupostos presentes na literatura de policy transfer. A forma escolhida para aplicar essas definições teóricas ocorreu por meio da análise empírica da temática da saúde dentro do Mercosul. Trata-se de um tema cuja importância e características possibilita a busca da cooperação entre os Estados, por meio de ações integradas que visam enfrentar doenças, epidemias ou lidar com critérios específicos para a realização da vigilância epidemiológica e controle sanitário entre as fronteiras nacionais. Neste artigo foi possível observar que as resoluções do GMC são, em sua essência, mais gerais do que específicas, ou seja, remetem a glossários de terminologias ou critérios básicos de inspeção sanitária. O contrário acontece com os temas presentes nos acordos RMS, que apresentam propostas de ações e planos integrados visando controlar e combater determinadas doenças, em particular a dengue e a AIDS.

A diferença da abordagem do tema da saúde dentro dos dois principais órgãos decisórios do Mercosul conduziu a estratégia de abordagem desenvolvida neste artigo. Conforme foi observado na seção precedente, a maior parte das resoluções do GMC precisava ser internalizada - 3 das 36 resoluções analisadas não requisitavam a internalização nos Estados nacionais. No caso dessas 
resoluções, foi essencial a análise do percurso que se inicia na identificação dos foros regionais específicos, com capacidade de produzir decisões, até o âmbito dos dois países aqui analisados (Brasil e Argentina), onde ocorreu a internalização das normas. O enfoque da pesquisa teve como ênfase a política de saúde e, mais especificamente, a área de trabalho da Vigilância Epidemiológica. Nesse sentido, ficou claro que as normativas provenientes do SGT 11, subordinado ao GMC, possuem características específicas que permitem essa análise, isto é, do modo como as resoluções aprovadas no GMC se tornam normas incorporadas pelos ministérios da saúde de cada Estado Parte do Mercosul.

Por fim, cumpre destacar que a pesquisa sobre transferência de políticas nos processos de integração regional precisa avançar com novos estudos empíricos, o que este artigo procurou fazer ao analisar o caso específico da internalização das resoluções aprovadas no GMC em dois protagonistas importantes do Mercosul - Brasil e Argentina.

*Artigo recebido em 05 de julho de 2019, aprovado em 10 de agosto de 2020.

\section{REFERÊNCIAS}

BULMER, Simon; PADGETT, Stephen. Policy transfer in the European Union: an institutionalism perspective. British Journal of Political Science, Cambridge University Press, v. 35, p. 103-126, jan. 2004.

CAETANO, Gerardo. "Breve historia del MERCOSUR en sus 20 años. Coyunturas e instituciones (1991-2011)" En: Gerardo Caetano (coord.): MERCOSUR 20 años. Montevideo: Centro de Formación para la Integración Regional CEFIR. 2011.

DOLOWITZ, David; MARSH, David. Learning from abroad: the role of policy transfer in contemporary policy-making. Governance: An International Journal of Policy and Administration, v. 13, n. 1, p. 5-24, Jan. 2000.

DOLOWITZ, David. Transfer and learning: One coin two elements. Novos estudos CEBRAP, São Paulo, v. 107, p. 35-56, Mar. 2017.

MACHADO, M. H.; PAULA, A. E.-K. de; AGUIAR Filho, W. O trabalho em saúde no Mercosul: Uma abordagem brasileira sobre a questão. Cadernos de Saúde Pública, Rio de Janeiro, 23, Sup. 2: p. 292-301, 2007.

MARIN, Pedro de Lima. Mercosul e a disseminação internacional de políticas públicas. Cadernos Gestão Pública e Cidadania, São Paulo, v. 16, n. 58, jan. 2011.

MEDEIROS, Marcelo de Almeida; MEUNIER, Isabel; COCKLES, Mariana. Processos de Difusão Política e Legitimidade no Mercosul: Mimetismo Institucional e Mecanismos de 
Internalização de Normas Comunitárias. Contexto Internacional, Rio de Janeiro, v. 37, n. 2, p. 537-570, 2015.

MERCOSUL. Resolução No 151/1996, de 14 de dezembro de 1996, criação do subgrupo de trabalho ${ }^{0} 11$ "Saúde". Disponível em: tinyurl.com/yxen34qa; Acesso em: 30/10/2019; 1996.

MERCOSUL. Tratado de Ouro Preto. Disponível em: tinyurl.com/y38otxrl; Acesso em: 30/10/2019; 2019a.

MERCOSUL. Organograma. Disponível em: tinyurl.com/y2bux3cm; Acesso em: 30/10/2019; 2019b.

MERCOSUL. Documentos. Disponível em: https://documentos.mercosur.int; Acesso em: 30/10/2019; 2019c.

MERCOSUL. Normativas dos órgãos decisórios do Mercosul. Disponível em: tinyurl.com/yxen34qa; Acesso em: 30/10/2019; $2019 \mathrm{~d}$.

OLIVEIRA, Osmany Porto de; FARIA, Carlos Aurélio Pimenta de. Policy transfer, diffusion, and circulation: Research Traditions and the State of the Discipline in Brazil. Novos estudos CEBRAP, São Paulo, v. 36, n. 1, p. 13-32, Mar. 2017.

PEREIRA, Alexsandro Eugenio; BERNARDO, Glaucia Julião; CULPI, Ludmila Andrzejewski; PESSALI, Huáscar Fialho. A governança facilitada no Mercosul: transferência de políticas e integração nas áreas de educação, migração e saúde. Revista de Administração Pública, 52(2), p. 285-302, 2018.

PEREIRA, Alexsandro Eugenio. Transferência e difusão de políticas públicas no Mercosul: Análise da formação de recursos humanos e do exercício profissional na área de saúde (1996-2014). Anais do 39 Encontro Anual da ANPOCS, Caxambú-MG, 2015.

SARAIVA, Miriam Gomes; ALMEIDA, Fernando Roberto de Freitas. A integração BrasilArgentina no final dos anos 90. Revista Brasileira de Política Internacional, Brasília, v. 42, n. 2, p. 18-39, Dec. 1999.

STONE, Diane. Learning Lessons and Transferring Policy across Time, Space and Disciplines. Politics, 19(1), p. 51-59, 1999.

STONE, Diane. Transfer Agents and Global Networks in the 'Transnationalisation' of Policy, Journal of European Public Policy, v. 11, n. 3, p. 545-566, jun. 2004.

VAZ, Alcides Costa. Cooperação, integração e processo negociador: A construção do Mercosul. Brasília: IBRI, 2002.

VENTURA, Deisy; ONUKI, Janina; MEDEIROS, Marcelo et al. Internalização das normas do MERCOSUL. Série Pensando o Direito, vol. 4. Brasília: Ministério da Justiça, 2012.

VIGEVANI, T.; FAVARON, G. de M.; RAMANZINI JÚNIOR, H.; CORREIA, R. A. O papel da integração para o Brasil: Universalismo, soberania e percepção das elites. Revista Brasileira de Política Internacional, Brasília, 51(1): 5-27, 2008. 
ZELICOVICH, Julieta. El MERCOSUR a 20 años del Protocolo de Ouro Preto: un balance de la dimensión comercial. Revista Latinoamericana de Desarrollo Económico, La Paz, n. 24, p. 97-120, nov. 2015. 